\title{
Editorial
}

\section{Wireless Cooperative Networks}

\author{
Andrea Conti,, Jiangzhou Wang, ${ }^{2}$ Hyundong Shin, ${ }^{3}$ Ramesh Annavajjala, ${ }^{4}$ and Moe Z. Win ${ }^{5}$ \\ ${ }^{1}$ Engineering Department in Ferrara (ENDIF), University of Ferrara, 44100 Ferrara, Italy \\ ${ }^{2}$ Department of Electronics, University of Kent, Canterbury, Kent CT2 7NT, UK \\ ${ }^{3}$ Department of Radio Communication Engineering, School of Electronics and Information, Kyung Hee University, \\ Gueonggi-Do 449-701, South Korea \\ ${ }^{4}$ Mitsubishi Electric Research Laboratories, 201 Broadway, Cambridge, MA 02139, USA \\ ${ }^{5}$ Department of Aeronautics and Astronautics, Massachusetts Institute of Technology, Cambridge, MA 02139, USA
}

Correspondence should be addressed to Andrea Conti, a.conti@ieee.org

Received 29 July 2008; Accepted 29 July 2008

Copyright (C) 2008 Andrea Conti et al. This is an open access article distributed under the Creative Commons Attribution License, which permits unrestricted use, distribution, and reproduction in any medium, provided the original work is properly cited.

Cooperative networks are gaining an increasing interest in information and communications technologies since such networks can improve communication capability and provide a fertile environment for the development of context-aware services. Cooperative communications and networking represent a new paradigm which involves both transmission and distributed processing, promising significant increase of capacity and diversity gain in wireless networks. From one hand, the integration of long-range and short-range wireless communication networks (e.g., infrastructured networks such as $3 \mathrm{G}$, wireless ad hoc networks, and wireless sensor networks) improves the performance in terms of both area coverage and quality of service (QoS). On the other hand, the cooperation among nodes, as in the case of wireless sensor networks, allows a distributed space-time signal processing which enables, among others, environmental monitoring, localization techniques, and distributed measurements, with a reduced complexity or energy consumption per node. The relevance of this topic is also reflected by numerous technical sessions in current international conferences as well as by the increasing number of national and international projects on these aspects.

This special issue aims to collect cutting-edge research achievements in this area. We solicited papers that present original and unpublished work on topics including, but not limited to, the following: physical layer models, for example, channel models (statistics, fading, MIMO, feedback); device constraints (power, energy, multiple access, synchronization) and resource management; distributed processing for cooperative networks (e.g., distributed compression in wireless sensor networks, channel and network codes design); performance metrics (e.g., capacity, cost, outage, delay, energy, scaling laws); cross-layer issues, for example, PHY/MAC/NET interactions, joint source-channel coding, separation theorems; multiterminal information theory; multihop communications; integration of wireless heterogeneous (long- and short-range) systems.

In "Asymptotic analysis of large cooperative relay networks using random matrix theory" by $\mathrm{H}$. Li et al., cooperative relay networks with large number of nodes are analyzed, and in particular the asymptotic performance improvement of cooperative transmission over direct transmission and relay transmission is analyzed using random matrix theory. The key idea is to investigate the eigenvalue distributions related to channel capacity and to analyze the moments of this distribution in large wireless networks. The analysis in this paper provides important tools for the understanding and the design of large cooperative wireless networks.

H. Van Khuong and T. Le-Ngoc propose, in the paper "Bandwidth-efficient cooperative relaying schemes with multi-antenna relay," coded cooperative relaying schemes in which all successfully decoded signals from multiple sources are forwarded simultaneously by a multiantenna relay to a common multiantenna destination to increase bandwidth efficiency. These schemes facilitate various retransmission strategies at relay together with single-user and multiuser iterative decoding techniques at destination, suitable for tradeoffs between performance, latency, and complexity.

The problem of choosing the best relay node in relaying networks is addressed in "Performance of multiple-relay cooperative diversity systems with best-relay selection over rayleigh fading channels" by S. S. Ikki and M. H. Ahmed. They consider an amplify-and-forward (AF) cooperative diversity system where a source node communicates with a 
destination node directly and indirectly (through multiple relays). It is shown that the best-relay selection reduces the amount of required resources while improving the performance. Authors derive closed form expressions for tight lower bounds on the symbol error probability and outage probability.

A double-differential modulation for the amplify-andforward protocol over Nakagami- $m$ fading channels with carrier offsets is proposed by M. R. Bhatnagar et al. in "Cooperative communications over flat fading channels with carrier offsets: a double-differential modulation approach." They propose an emulated maximum ratio combining (EMRC) decoder, which could be used by the doubledifferential receiver in the absence of exact channel knowledge. Approximate bit error rate (BER) analysis is performed for the double-differential modulation-based cooperative communication system. They propose a double-differential system which is immune to random carrier offsets, whereas the conventional single-differential modulation-based cooperative system breaks down, and perform better than training-based cooperative system which utilizes training data to estimate carrier offsets and channel gains.

In "Delay optimization in cooperative relaying with cyclic delay diversity," S. B. Slimane et al. propose to inserting random delays at the nonregenerative fixed relays to further improve the system performance. However, random delays result in limited performance gain from multipath diversity. In this paper, two promising delay optimization schemes are introduced for a multicellular OFDM system with cooperative relaying, stationary multiple users, and fixed relays.

A. Conti, V. Tralli, and M. Chiani address the construction of space-time codes for cooperative communications over block fading channels in the paper "Pragmatic spacetime codes for cooperative relaying in block fading channels." They consider a pragmatic approach based on the concatenation of convolutional codes and binary/quaternary phase shift keying modulation to obtain cooperative codes for relay networks. The pairwise error probability, an asymptotic bound on the frame error probability, and a design criterion to optimize both diversity and coding gain are also derived. While the implementation of pragmatic space-time codes only requires common convolutional encoders and Viterbi decoders with suitable generators, rate, and branch metric, they perform well in block fading channels, including quasistatic channel, even with a low number of states and relays.

In "Interference mitigation in cooperative SFBCOFDM," D. Sreedhar and A. Chockalingam consider cooperative space-frequency block-coded orthogonal frequencydivision multiplexing (SFBC-OFDM) networks with amplify-and-forward (AF) and decode-and-forward (DF) protocols at the relays. They propose an interference cancellation algorithm for this system at the destination node, and show that the proposed algorithm effectively mitigates the intersymbol interference and intercarrier interference effects.

For what concerns the MAC layer, J. Alonso-Zrate et al., in "Persistent RCSMA: a MAC protocol for a distributed cooperative ARQ scheme in wireless networks," present the persistent relay carrier sensing multiple access (PRCSMA) protocol that allows for the execution of a distributed cooperative automatic retransmission request (ARQ) scheme in IEEE 802.11 wireless networks. The underlying idea of the PRCSMA protocol is to modify the basic rules of the IEEE 802.11 MAC protocol to execute a distributed cooperative ARQ scheme in wireless networks to enhance their performance and to extend coverage.

At the scheduling level, "Optimally joint subcarrier matching and power allocation in OFDM multihop system" by W. Wang et al. propose an optimally joint subcarrier matching and power allocation scheme to maximize the channel capacity under total system power constrain of OFDM systems. The problem is formulated as a mixed binary integer programming problem (which is prohibitive to find the global optimum in terms of complexity) and then a low-complexity scheme by making use of the equivalent channel power gain for any matched subcarrier pair is proposed.

W. Mesbah and T. N. Davidson study, in "Power and resource allocation for orthogonal multiple access relay systems," the problem of joint power and channel resource allocation for orthogonal multiple access relay (MAR) systems to maximize the achievable rate region. The authors consider four relaying strategies and show that the problem can be formulated as a quasiconvex problem in several cases. Therefore, efficient algorithms can be derived for joint optimal power and channel resource allocation.

At networking level, the paper "Resource sharing via planed relay for HWN" by C. Shen et al. presents an improved version of adaptive distributed cross-layer routing algorithm for hybrid wireless network with dedicated relay stations. They verify that the performance of routing protocol benefits of the hybrid wireless networks nature.

Collaboration in heterogeneous wireless networks is addressed by A. Bazzi et al. a in "Multi radio resource management: parallel transmission for higher throughput." Mobile communication systems beyond the third generation will see the interconnection of heterogeneous radio access networks (UMTS, WiMax, wireless local area networks, etc.) to always provide the best QoS to users with multimode terminals. The issue of parallel transmission over multiple radio access technologies (RATs) is investigated focusing the attention on the QoS perceived by the end users. It shows the real benefit of parallel transmission over multiple RATs and how it is conditioned to the fulfilment of some requirements related to the particular kind of RATs, the multiradio resource management strategy, and the transport level protocol behavior.

Andrea Conti Jiangzhou Wang Hyundong Shin Ramesh Annavajjala Moe Z. Win 